\title{
Urban Wetland Change Detection Using Time-Series Remote Sensing Data
}

\author{
Lin $\mathrm{Liu}^{1,2}$, Yapeng Zhou ${ }^{3}$, Li Wang ${ }^{4, *}$, Jianchun $\mathrm{Hou}^{5}$, and Mingquan $\mathrm{Wu}^{4}$ \\ ${ }^{1}$ School of Land Science and Technology, China University of Geosciences, \\ Beijing 100083, China \\ ${ }^{2}$ Shijiazhuang Engineering Technology School, Shijiazhuang 050061, China \\ ${ }^{3}$ College of Natural Resources \& Environment Sciences, \\ Agricultural University of Hebei, Baoding 071001, China \\ ${ }^{4}$ State Key Laboratory of Remote Sensing Science, \\ Jointly Sponsored by the Institute of Remote Sensing Applications of Chinese \\ Academy of Sciences and Beijing Normal University, Beijing 100101, China \\ ${ }^{5}$ China National Petroleum Corporation, 100007 Beijing, China \\ Liulin7801@126.com, Wangli@irsa.ac.cn
}

\begin{abstract}
Wetland, as one of the weakest ecosystems in the world, is deteriorating rapidly in many regions. Longfeng wetland is the biggest urban wetland in China, located in the inner of Daqing city, Heilongjiang Province. It is divided into two areas by Wolong road, the east is provincial natural reserve and the west is unprotected. This paper describes a remote sensing change detection approach used to assess Longfeng wetland change. Four images were used, i.e., Landsat MSS in August 1979, Landsat TM in July 1990 and in August 2001, LISS-III in July 2006. Linear spectral mixture model (LSMM) method was used to classify the wetland and the results were satisfying. The results showed that the land cover types of Longfeng wetland had been greatly affected by human civilities (such as city extension and fishery), which was more deeply in unprotected area than in natural reserve.
\end{abstract}

Keywords: Change detection, Remote sensing, Time-series images, Urban wetland.

\section{$1 \quad$ Introduction}

Wetlands account for only three to six percent of the earth's land surface, but they provide service of great value to human beings, for example, they can control flood, protect coastal zones, replay water supplies, improve water quality, produce agricultural product, and provide plant and wildlife habitat [1,2,3]. Despite these proven advantages, wetlands have been one of the weakest ecosystems in the world. In many regions wetlands are deteriorating rapidly $[3,4]$.

The Longfeng wetland is located in the inner of Daqing city, Heilongjiang Province of China (figure 1). It is the biggest urban wetland in China. It is divided into two areas

\footnotetext{
* Corresponding author.

D. Li and Y. Chen (Eds.): CCTA 2012, Part II, IFIP AICT 393, pp. 177-186, 2013.

(C) IFIP International Federation for Information Processing 2013
} 
by Wolong road, the east is provincial natural reserve and the west is unprotected. Daqing city is the largest petrochemical industry base in China. Over the last two decades (1985-2005), Daqing has undergone a high-speed economic development and environmental deterioration as well.

The objective of the present study was to monitor the changes of Longfeng wetland using multi-temporal and multi-source remote sensing data, to understand its change patterns, and to analyze the relationships between natural and human factors and the deterioration of Longfeng wetland. To achieve this goal, the following 3 objectives were presented:

1) Choose appropriate methods to classify these four multi-temporal images, and evaluate the classification accuracy;

2) Monitor Longfeng wetland dynamics using four classified remote sensing images;

3) Study the urban wetland dynamics; analyze the relationship between natural and human factors and these changes.

\section{Study Site}

The printing area is $122 \mathrm{~mm} \times 193 \mathrm{~mm}$. The text should be justified to occupy the full line width, so that the right margin is not ragged, with words hyphenated as appropriate. Please fill pages so that the length of the text is no less than $180 \mathrm{~mm}$, if possible.

Covering an area of 8,000 ha, the Longfeng wetland is located in the middle of Songnen Plain in Heilongjiang Province in the northeast of China, between $46^{\circ} 35^{\prime}-$ $46^{\circ} 41^{\prime} \mathrm{N}$ latitudes and $125^{\circ} 20^{\prime}-125^{\circ} 26^{\prime} \mathrm{E}$ longitudes(figure 1). The study area has typical characteristics of continental monsoon climate.

Longfeng wetland is encompassed by two districts of Daqing city, i.e., Longfeng and Wolitun, traversed by Wolong road and Binzhou railway, approximately $8 \mathrm{~km}$ from the centre of Daqing city. It is very helpful to improve and maintain ecological system and environment of Daqing city. However, the fast development of Daqing city leads to the rapid reduction and deterioration of the Longfeng wetland.

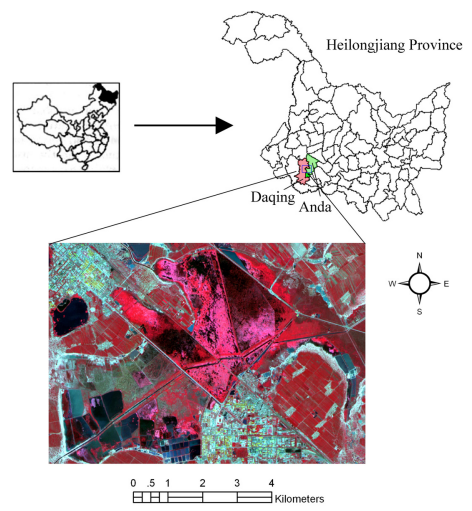

Fig. 1. Location of Longfeng wetland in Heilongjiang province, China 


\section{Methodology}

In our papers, one hybrid classification approach was used. Firstly, based on the linear spectral mixture model (LSMM), the imagery was transformed into corresponding end-member bands (including water body, bare land, and green vegetation). In the result of linear spectral unmixing, three classes, i.e. water body, bare land and green vegetation, can be easily extracted, because the pixel values of these three classes are highest in corresponding band. To make change detection, only three classes are not far enough. According to the characteristics of Longfeng wetland, the imagery would be classified into 6 classes, and the ranges of corresponding pixel value of the 6 classes were shown in table 3 . Through comparing the value of 3 end-member bands of each pixel, the 6 classes were very easily classified.

\subsection{LSMM Method}

Linear spectral mixture model is based on the idea that a continuous scene can be modeled as the sum of the radiometric interactions of individual cover types weighted by their relative proportions [5]. Mathematically, the linear spectral mixture analysis framework can be expressed as follows:

$$
\begin{gathered}
R_{i}=\sum_{j=1}^{n} f_{j} R_{j}+\varepsilon_{i} \\
0 \leq f_{j} \leq 1 \text { and } 0 \leq \sum_{j=1}^{n} f_{j} \leq 1
\end{gathered}
$$

Where $R_{i}$ is the composite reflectance of the mixed spectrum in band i, $f$ is the weight of end-member $(i)$ in the mixture, $R_{j}$ is the reflectance of that end-member in band $j, j$ is the number of end-members, $\varepsilon_{i}$ is the error in the sensor band i. Eq.(3) constraints the weight and the sum of the weight is allowed to be between 0 to 1 .

According to these formulas the value of a pixel must equal the sum of the values of the end-members times their abundance. The fit of the model can be assessed by the root mean square error (RMSE):

$$
R M S E=\sqrt{\frac{\sum_{i=1}^{n} \varepsilon_{i}^{2}}{n}}
$$

Where $i$ is the number of bands used in the spectral unmixing. Higher values of RMSE indicate regions that could contain lacking end-members.

\subsection{End-Members Selection}

The key to a successful mixture model is the selection of appropriate end-members $[6,7]$. These end-members principally being pure reflectance spectra that are derived by 
a specific target material with no mixing with any other materials are usually selected either from spectral libraries built from field surveys [8] or from the image data.

In Longfeng wetland, bare land (BL), water body (WB) and green vegetation (GV) were chosen to represent the spectral variability. In order to successfully complete the spectral unmixing, it was very essential to accurately estimate the spectral endmembers for each component of 3 end-members. These end-members were determined from the two-dimensional scatter plot of RED and NIR bands. In order to get the purest pixels, the MNF transformation was employed to the four images, and then PPI was computed. Those pixels with PPI great than 10 were extracted and projected to the two-dimension of RED and NIR bands. The PPI images and twodimensional scatter plot were shown in figure 2. Figure 2 (b) showed that rather symmetrical patterns in pixel distributions allowed the appropriate identification of three dominant spectral end-members, bare land (BL), green vegetation (GV) and water body (WB), at the extremes of image feature space of red (MSS B2, LISS-III
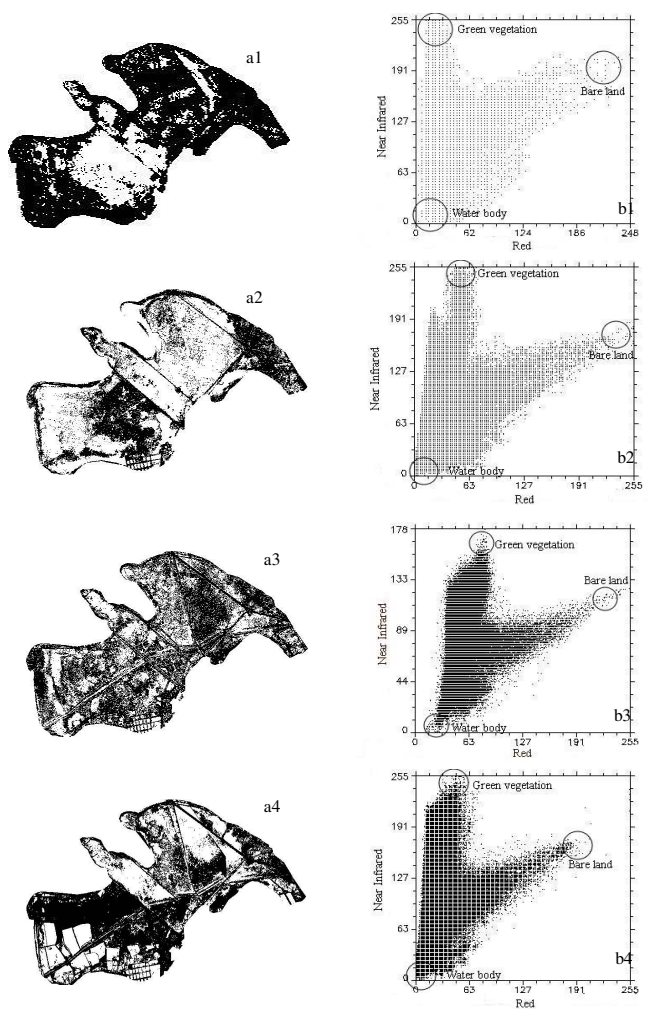

Fig. 2. PPI images (a) and the corresponding two-dimensional scatter plots of red and NIR bands(b). a1, a2, a3 and a4 denote 1979 MSS, 1990 TM, 2001 TM and 2006 LISS-III image. In b1, red band is MSS B2 and NIR is MSS B4; In b2 and b3, red band is TM B3 and NIR is TM B4; In b4, red band is LISS-III B2 and NIR is LISS-III B3. End-members derive from b1, b2, b3 and b4. 
B2, TM B3) and NIR (MSS B4, LISS-III B3, TM B4) bands. An average of pixels of these vertices were calculated and used in LSMM. The BL end-member was identified at the top right vertex while GV end-member on the left vertex of the scatter plots. The WB end-member was selected from the pixels at the origin of the scatter plots.

End-member spectra were shown in figure 3, and used in the LSMM. After using image normalization and the same end-member selection method, the end-member spectra of 4 dates were very similar. Therefore, using these end-member spectra to analyze 4 scenes assured that the 4 LSMM results had more comparability.

(a)

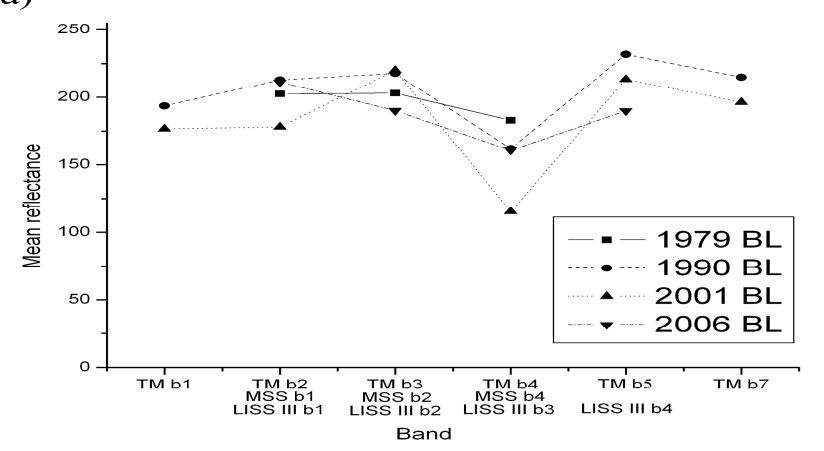

(b)

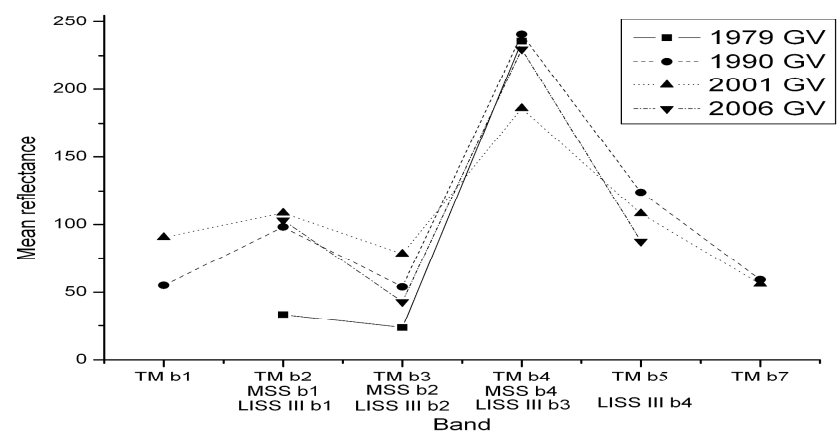

(c)

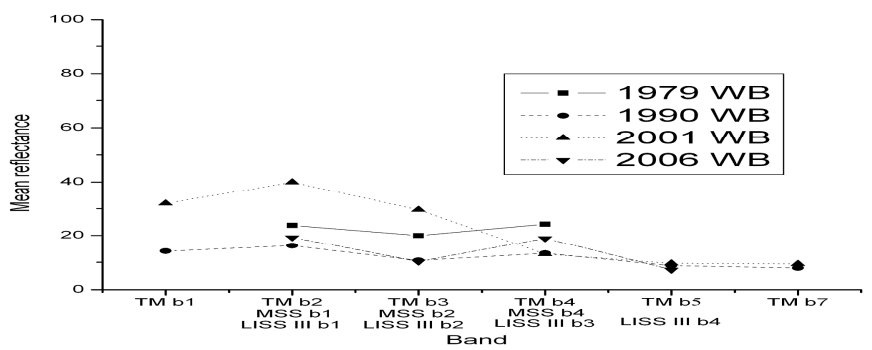

Fig. 3. End-member spectra used in LSMM. These end-member spectra were respectively derived from 4 temporal images. (a) is the spectra of bare land (BL); (b) is the spectra of green vegetation $(\mathrm{GV})$; (c) is the spectra of water body $(\mathrm{GV})$. 


\subsection{LSMM Result}

The primary results of LSMM were separate images for each of the end-members (BL, GV and WB) containing an estimate of the percentage of that end-member in each pixel. When the model was run, each pixel value corresponded to the fraction of $\mathrm{BL}, \mathrm{GV}$ and $\mathrm{WB}$ in that pixel and the range of fraction values was between 0 and 1 . Figure 4 illustrated the fraction images of the 4 dates.

After achieving LSMM results of the 4 date's images, the six classes were identified in LSMM results. Some samples of 6 classes were extracted from the 2006 image, based on the investigated ground truth information and GPS location. Then the characteristics of 6 classes were obtained from 2006 image. According to these characteristics, other dates' samples of 6 classes were chosen, and the spectral curve of 6 classes at each temporal were extracted. Figure 5 is the spectral curve of 6 classes at the end-member band in 2006 LSMM result.

Table 1. The classification algorithm

\begin{tabular}{lcc}
\hline \multicolumn{1}{c}{ Classes } & Algorithm* & Step \\
\hline Bare land and construction (BLC) & $\mathrm{BL}>1$ & 1 \\
& & \\
Dense reed (DR) & $\mathrm{GV}>1$ & 1 \\
Open water (include fishpond) (OW) & $\mathrm{WB}>1$ & 2 \\
Sparse reed (SR) & $\mathrm{BL}<\mathrm{WB} \& \mathrm{BL}<\mathrm{GV}$ & 2 \\
Dry grassland (DG) & $\mathrm{WB}<\mathrm{BL} \& \mathrm{WB}<\mathrm{GV}$ & 2 \\
Muddy area (MA) & $\mathrm{GV}<\mathrm{BL} \& \mathrm{GV}<\mathrm{WB}$ & 2 \\
\hline
\end{tabular}

* 'BL', 'GV' and 'WB' represent the pixel value normalized in corresponding band.

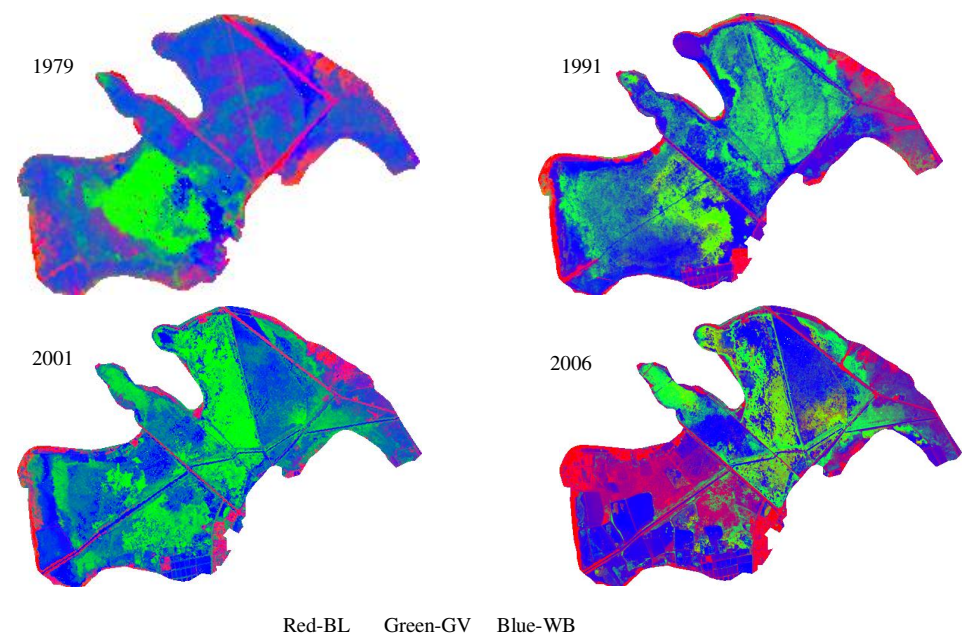

Fig. 4. Fraction images for three end-members, bare land (BL) in red, green vegetation $(\mathrm{GV})$ in green and water body (WB) in blue, obtained by LSMM 


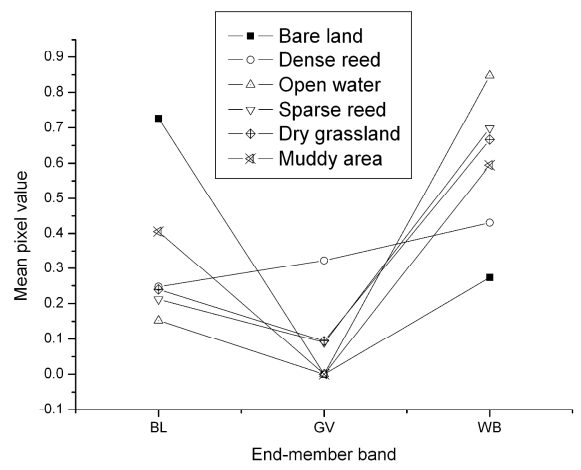

Fig. 5. Mean curves of 6 classes at the end-member band in 2006 LSMM result

\section{$4 \quad$ Results and Discussion}

\subsection{Land Cover Change Result}

We had only survey data in 2006, and land types in wetland changed greatly, so only the error in 2006 was analyzed. Because of the consistent classification method, classification accuracy of other years cannot be low. Error matrices were used to assess classification accuracy, shown in table 5 for 2006. The overall accuracy for 2006 was $90.49 \%$, with Kappa statistics of $88.57 \%$. User and producer accuracies of individual classes were consistently high, ranging from $75 \%$ to $100 \%$.(Table 4 )

Table 2. The error matrices of classification for 2006

\begin{tabular}{|c|c|c|c|c|c|c|c|c|}
\hline Class & BLC & $\mathrm{DR}$ & OW & MA & SR & DG & Total & User Acc. \\
\hline BLC & 171 & 0 & 0 & 0 & 0 & 0 & 171 & 100 \\
\hline DR & 0 & 218 & 0 & 0 & 23 & 34 & 275 & 79.27 \\
\hline ow & 0 & 0 & 181 & 0 & 0 & 0 & 181 & 100 \\
\hline MA & 1 & 0 & 0 & 198 & 26 & 0 & 225 & 88 \\
\hline SR & 0 & 1 & 30 & 0 & 153 & 0 & 184 & 83.15 \\
\hline DG & 0 & 0 & 0 & 0 & 0 & 173 & 173 & 100 \\
\hline Total & 172 & 219 & 211 & 198 & 202 & 207 & 1209 & \\
\hline $\begin{array}{l}\text { Prod. } \\
\text { Acc. }\end{array}$ & 99.42 & 99.54 & 85.78 & 100 & 75.74 & 83.57 & & \\
\hline \multicolumn{9}{|c|}{ Overall Accuracy $=90.49 \%$} \\
\hline Kappa & ficient & & & & & & & \\
\hline
\end{tabular}


Classification maps were generated for all four years (figure 4) and the individual class area and change statistics for the four years were summarized in table 6 .

From 1979 to 2006, bare land and construction land increased approximately 500 ha, and open water increased 850 ha while muddy area decreased 1650 ha. Relatively, bare land and construction land increased $185.20 \%$ from 1979 to 2006, with the greatest increase occurring from 1990 to 2001; open water increased $494.41 \%$, while muddy area decreased $57.36 \%$. Compared to the changes with the former mentioned three land uses, dense reed, sparse reed and dry grassland changed slightly between 1979 and 2006. In 1979, the proportion of these three classes was, respectively, $15.37 \%, 24.18 \%$ and $19.19 \%$ and changed to $18.87 \%, 27.31 \%$ and $16.77 \%$ in 2006 . The variations were only $3.5 \%, 3.13 \%$ and $2.42 \%$.

To further evaluate the results of land cover conversions, matrices of land cover changes from 1979 to 1990,1990 to 2001, and 2001 to 2006 were created (Table 5). In the table, unchanged percentages were located along the major diagonal of the matrix. These results indicated that the muddy area had the most variation with the least unchanged percentage in three periods, respectively, 12, 0 and 14. From figure 6, we also found this great change. For instance, highlighted area a (a1 and a2) in figure 6 , indicated the change process $\mathrm{MA} \rightarrow \mathrm{BLC} ; \mathrm{b}$ (b1 and b2) showed the process $\mathrm{MA} \rightarrow \mathrm{DR}$ or $\mathrm{SR} \rightarrow \mathrm{OW}$; $\mathrm{c}$ illustrated $\mathrm{MA} \rightarrow \mathrm{DG}$, and $\mathrm{d}$ was $\mathrm{MA} \rightarrow \mathrm{BLC} \rightarrow \mathrm{MA}$.

Table 5 showed the BLC was stable with almost highest unchanged percentage in three periods. This stabilization owed to the construction in BLC, which supported by highlighted a (figure 6). In highlighted area a, approved by field survey, a1 was the urban expansion of Wolitun, and a2 was a fishing workshop. Highlighted area showed if the MA were changed to BLC then the changed area were not transition but only expanded. Nevertheless, we also found the BLC conversion to other classes. In the study area, bare land was formed as a result of salinization, and its variation had close relation with rainfall. A example is highlighted area d (figure 6), showing the process $\mathrm{MA} \rightarrow \mathrm{BLC} \rightarrow$ MA.

Table 3. Transition between the major land use types (area in \%) in the Longfeng wetland

\begin{tabular}{lrrrrrr}
\hline Land use type & BLC & DR & OW & MA & SR & DG \\
\hline 1979-1990 & & & & & & \\
BLC & $\mathbf{3 8}$ & 2 & 24 & 16 & 8 & 8 \\
DR & 5 & $\mathbf{2 1}$ & 15 & 7 & 44 & 7 \\
OW & 0 & 35 & $\mathbf{4 3}$ & 1 & 20 & 1 \\
MA & 7 & 11 & 27 & $\mathbf{1 2}$ & 33 & 10 \\
SR & 1 & 26 & 21 & 2 & $\mathbf{4 4}$ & 5 \\
DG & 3 & 28 & 22 & 6 & 35 & $\mathbf{6}$ \\
1990-2001 & & & & & & \\
BLC & $\mathbf{8 2}$ & 0 & 1 & 0 & 4 & 12 \\
DR & 1 & $\mathbf{3 8}$ & 2 & 7 & 51 & 2 \\
OW & 10 & 17 & $\mathbf{1 2}$ & 7 & 48 & 6 \\
\hline
\end{tabular}


Table 3. (Continued)

\begin{tabular}{lrrrrrr}
\hline MA & 40 & 2 & 7 & $\mathbf{0}$ & 19 & 31 \\
SR & 3 & 28 & 3 & 10 & $\mathbf{5 4}$ & 2 \\
DG & 17 & 7 & 1 & 0 & 25 & $\mathbf{4 8}$ \\
2001-2006 & & & & & & \\
BLC & $\mathbf{4 7}$ & 2 & 10 & 14 & 9 & 15 \\
DR & 1 & $\mathbf{4 7}$ & 7 & 13 & 24 & 8 \\
OW & 9 & 5 & $\mathbf{5 5}$ & 13 & 16 & 2 \\
MA & 2 & 8 & 23 & $\mathbf{1 4}$ & 50 & 3 \\
SR & 4 & 16 & 11 & 17 & $\mathbf{3 7}$ & 15 \\
DG & 6 & 3 & 5 & 12 & 6 & $\mathbf{6 7}$ \\
\hline
\end{tabular}

\section{Conclusion}

Longfeng wetland located in Daqing city is one of the biggest wetlands in China. Because of the fast development of Daqing city, this wetland had been destroyed and occupied to some extent. This paper analyzed quantitatively the change pattern of Longfeng wetland using multi-temporal remote sensing data. This research aimed to explore the long-term dynamics in land cover and the change driving forces in Longfeng wetland, and revealed that there were substantial land use changes in the area from 1979 to 2006 . General patterns and trends of land use change in the area were evaluated by: (1) classifying images used LSMM method and get satisfying result; (2) comparing the classified results of 4 dates; (3) analyzing the major changes in the whole area and these changes in relation to natural and human factors. In addition, the different change patterns between protected area and unprotected area were compared, which indicated the influence of human activities on this area and the protection function of natural reserve.

The drawback of this research is that there are not so many classes, owing to the limits of remote sensing image. Especially, caused by spectral similarity, it is very difficult to separate bare land into construction and water into rearing pond, which affects the correlation analysis between changes and human activity. However, in this research, according to the field survey result and the special imagery characteristics (there are many roads between ponds or constructions), the influence is minimized.

This study showed that the ecosystem of Longfeng wetland had been threatened by the rapid development of Daqing city. In unprotected area, many areas had been filled with rearing pond and urban expansion. However, in natural reserve, the wetland had been protected very well, the areas of dense reeds had consistently increased, and the urban expansion did not occupy the areas of natural reserve (figure 8). Therefore, establishment of natural reserve is a good method to prevent the degradation and improve the ecosystem of wetland. 
Acknowledgments. We would like to appreciate the constructive suggestions by both reviewers and editor, which made the article more consistent. This work was funded by an NSFC grant (41001209) and China's Special Funds for Major State Basic Research Project (2010CB950603).

\section{References}

1. Acreman, M.C., Hollis, G.E.: Water management and wetlands in Sub-Saharan African. IUCN, Gland (1981)

2. Kashaigili, J.J., Mbilinyi, B.P., Mccartney, M., Mwanuzi, F.L.: Dynamics of Usangu plains wetlands: Use of remote sensing and GIS as management decision tools. Physics and Chemistry of the Earth 31, 15-16 (2006)

3. Sugumaran, R., Harken, J., Gerjevic, J.: I Using Remote Sensing Data to Study Wetland Dynamics in Iowa. In: Iowa's 6th Biennial GIS Conference, Ames, IA (2003)

4. http://www. wetlands.org/

5. Graetz, R.D.: Remote Sensing of Terrestrial Ecosystem Structure: An Ecologist 's Pragmatic View. Remote Sensing of Biosphere Functioning. Ecological Studies 77, 5-30 (1990)

6. Elmore, A.J., Mustaro, J.F., Manning, S.J., Lobell, D.B.: Quantifying Vegetation change in semiarid environments: Precision and accuracy of Spectral Mixture analysis and the Normalized Difference Vegetation index. Remote Sensing of Environment 73(1), 87-102 (2000)

7. Shanmugama, P., Ahna, Y.-H., Sanjeevib, S.: A comparison of the classification of wetland characteristics by linear spectral mixture modelling and traditional hard classifiers on multispectral remotely sensed imagery in southern India. Ecological Modelling 194(4), 379-394 (2006)

8. Asner, G.P., Heidebrecht, K.B.: Spectral unmixing of vegetation, soil and dry carbon cover in arid regions: comparing multispectral and hyperspectral observations. International Journal of Remote Sensing 23(19), 3939-3958 (2002) 